\title{
Spontaneous Uterine Perforation Secondary to Leiomyosarcoma Arising in a Uterine Leiomyoma
}

\author{
Kyousuke Takeuchi*, Makoto Sugimoto, Ai Yoshida, Shino Yamashita, Taro Tsujino, \\ Riichiro Nishino \\ Department of Obstetrics and Gynecology, Kobe Medical Center, Kobe, Japan \\ Email: ${ }^{*}$ kyousuket@dolphin.ocn.ne.jp
}

Received 23 October 2013; revised 20 November 2013; accepted 28 November 2013

Copyright (C) 2014 by authors and Scientific Research Publishing Inc.

This work is licensed under the Creative Commons Attribution International License (CC BY).

http://creativecommons.org/licenses/by/4.0/

(c) (;) 0pen Access

\begin{abstract}
Histogenesis of uterine leiomyosarcoma has been controversial. It is generally believed that uterine leiomyosarcomas arise de novo, rather than from any precursor lesions. We report an unusual case of spontaneous rupture of leiomyosarcoma arising in pre-existing calcified uterine leiomyoma, which has been monitored over the years after menopause. This case also suggests that spontaneous intraperitoneal hemorrhage associated with malignant transformation of uterine leiomyoma, although rare, should be considered in postmenopausal women presenting with rapid growing uterine mass.
\end{abstract}

Keywords

Uterine Leiomyosarcoma; Leiomyoma; Malignant Transformation; Spontaneous Rupture

\section{Introduction}

Uterine leiomyosarcoma is an aggressive soft tissue neoplasm occurring in women of all ages, usually presenting with pelvic pain, mass and vaginal bleeding [1] [2]. Rarely, the presenting manifestations are related to hemoperitoneum caused by tumor rupture. The pathogenesis of uterine leiomyosarcoma is controversial. Although most cases of uterine leiomyosarcomas have been thought to arise de novo, several cases of malignant transformation of uterine leiomyoma have been reported [3]-[5]. We herein report an unusual case of hemoperitoneum caused by spontaneous rupture of rapid growing leiomyosarcoma arising in pre-existing leiomyoma of the uterus, which has been monitored over the years.

\footnotetext{
${ }^{*}$ Corresponding author.
} 


\section{Case}

A 59-year-old postmenopausal woman, para 2, gravida 2, presented deteriorating abdominal distention and pain. She had been annually submitted to transvaginal ultrasound examination for an intramural leiomyoma with calcification (4 cm in diameter) for four years. No alteration visible through ultrasound was detected in the lesion. The patient had no history of use of oral contraceptives or hormone replacement therapy. She was nauseated but denied vomiting or having constipation, abdominal trauma or exertion. A diffusely tender abdomen and a nontender midline abdominal mass was palpated. Vaginal ultrasound confirmed an enlarged uterine mass with calcification in it and the presence of moderate free abdominal fluid. Magnetic resonance (MR) imaging revealed the hemoperitoneum and the uterus to be significantly enlarged with a heterogeneous tumor measuring $11 \times 11$ $\times 8 \mathrm{~cm}$ with calcification inside the mass (Figure 1). The patient's situation deteriorated rapidly and signs of hypovolemic shock occurred. She subsequently underwent exploratory laparotomy, which revealed hemoperitoneum with approximately $700 \mathrm{ml}$ of old blood from a uterine fundal perforation (Figure 2). The uterus was bulky, but with no obvious extrauterine metastasis. Hysterectomy and bilateral salpingo-oophorectomy were performed. The myometrium contained an 11-cm mass with rupture through the uterine body. The center of the mass contained a 4-cm calcification. The endometrium was grossly normal. The reminder of the myometrium was unremarkable. Histologically, the features of the portions surrounding partly calcified mass were compatible with leiomyosarcoma (more than 10 mitoses per $10 \mathrm{HPF}$ ) with severe nuclear atypical and vascular space involvement. She received adjuvant treatment with four courses of docetaxel and gemcitabine hydrochloride administration. She is alive and well with no evidence of disease 16 months after surgery.

\section{Discussion}

Most uterine smooth muscle neoplasms are benign leiomyomas and leiomyosarcomas account for only $0.1 \%$ of uterine smooth muscle tumors [6] and 3\% to 7\% of uterine cancers [7]. Most occur in women over 40 years of age who usually present with vaginal bleeding, palpable pelvic mass, and pelvic pain. Malignancy should be suspected by the presence of certain clinical behaviors, such as tumor growth in menopausal women. Spontaneous rupture of uterine leiomyosarcoma in our case is an unusual cause of hemoperitoneum, which may be catastrophic if it is not promptly diagnosed and treated.

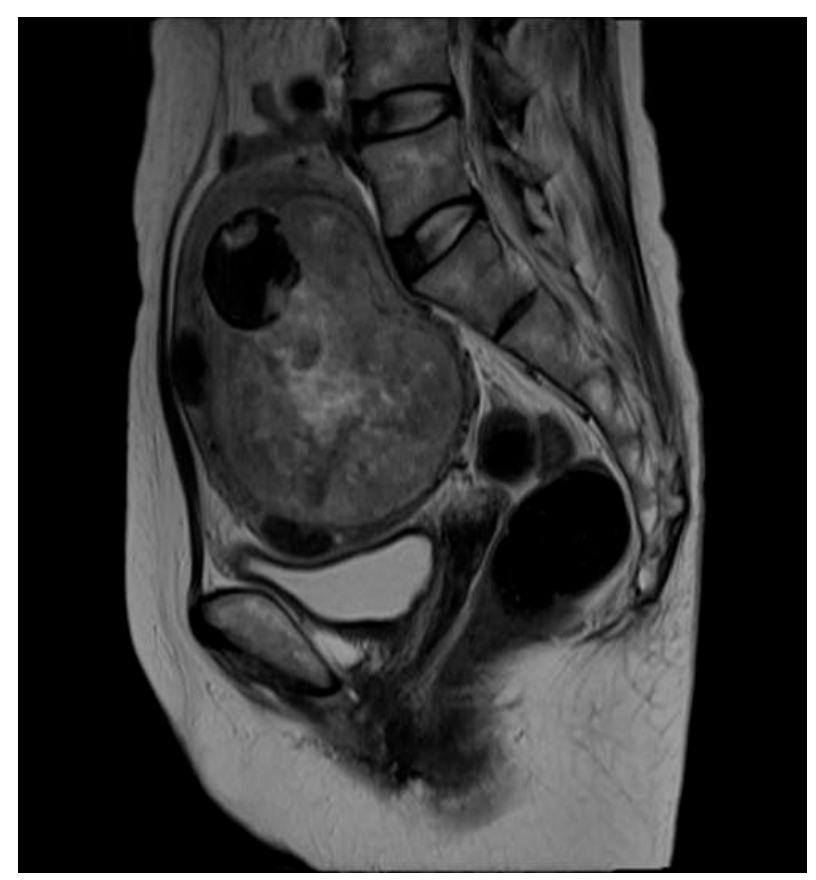

Figure 1. MR imaging showing significantly enlarged uterus with a heterogeneous tumor measuring $11 \times 11 \times 8 \mathrm{~cm}$ with calcification inside the mass. 


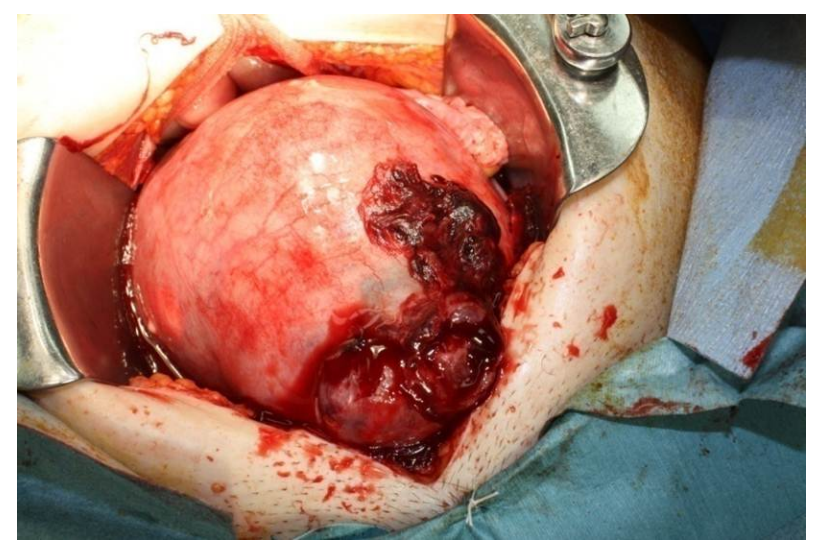

Figure 2. Uterine perforation secondary to leiomyosarcoma.

The pathogenesis of uterine leiomyosarcoma has been controversial. It is generally believed that uterine leiomyosarcomas arise de novo, rather than from any precursor lesions. However, there have been occasional reported examples of leiomyosarcomatous transformation in a leiomyoma. Mittal et al. [5] reported that about half of uterine leiomyosarcoma were associated with leiomyoma and they also showed cytogenetic evidence of the common origin of the leiomyosarcoma and leiomyoma components in the cases of leiomyosarcoma with in leiomyomatous area. Yanai et al. [3] recently reported leiomyoma associated with leiomyosarcoma are not restricted to cellular or bizarre leiomyoma and ordinary leiomyoma can also develop leiomyosarcoma. They also indicate that even small tumors have potential to transform.

\section{Conclusion}

Our case illustrates that malignant transformation of leiomyoma can occur even in small calcified leiomyoma, which is believed to be much quieter than the usual leiomyoma.

Thus, physicians should keep in mind the possible existence of malignancies arising even in calcified leiomyomas in postmenopausal women.

\section{Conflict of Interest}

The authors have no conflicts of interest in connection with submitted material.

\section{Content}

We report an unusual case of spontaneous rupture of leiomyosarcoma arising in pre-existing calcified uterine leiomyoma after menopause.

\section{References}

[1] Hicks, G., McCallum, I.J., Ogah, K., Guirguis, M. and Kasaraneni, R. (2010) Spontaneous Uterine Perforation Secondary to Uterine Leiomyosarcoma Presenting as Acute Abdomen with Haemoperitoneum. Journal of Obstetrics \& Gynaecology, 30, 211-212. http://dx.doi.org/10.3109/01443610903477523

[2] Farhi, J., Dicker, D. and Ben-Rafael, Z. (1993) Leiomyosarcoma Presenting as Spontaneous Hemoperitoneum. International Journal of Obstetrics \& Gynaecology, 40, 249-450. http://dx.doi.org/10.1016/0020-7292(93)90839-O

[3] Yanai, H., Wani, Y., Notohara, K., Takada, S. and Yoshino, T. (2010) Uterineleiomyosarcoma Arising in Leiomyoma: Clinicopathological Study of Four Cases and Literature Review. Pathology International, 60, 506-509. http://dx.doi.org/10.1111/j.1440-1827.2010.02549.x

[4] Indraccolo, U., Luchetti, G. and Indraccolo, S.R. (2008) Malignant Transformation of Uterine Leiomyomata. European Journal of Gynaecological Oncology, 29, 543-544.

[5] Mittal, K. and Joutovsky, A. (2007) Areas with Benign Morphologic and Immunohistochemical Features Are Associated with Some Uterine Leiomyosarcomas. Gynecologic Oncology, 104, 362-365.

http://dx.doi.org/10.1016/j.ygyno.2006.08.034 
[6] Zaloudek, C. and Hendrickson, M.R. (2001) Mesenchymal Tumors of the Uterus. In: Kuman, R.J., Ed., Blaustein’s Pathology of Female Genital Tract. 5th Edition, Springer-Verlag, New York, 561-615.

[7] Major, F.J., Blessing, J.A., Silverberg, S.G., Morrow, C.P., Creasman, W.T., Currie, J.L., Yordan, E. and Brady, M.F. (1993) Prognostic Factors in Early Stage Uterine Sarcoma: A Gynecologic Oncology Group Study. Cancer, 71, $1702-$ 1709. http://dx.doi.org/10.1002/cncr.2820710440 